Boletín de la Sociedad Geológica Mexicana

VOLUMEN 63, NÚM. 3, 2011, P. 429-443

\title{
Placas columnares de crinoides del Carbonífero de la región de Santiago Ixtaltepec, Municipio de Nochixtlán, Oaxaca; consideraciones estratigráficas y paleobiogeográficas
}

\author{
Rafael Villanueva-Olea ${ }^{1, *}$, Karla María Castillo-Espinoza ${ }^{1}$, Francisco Sour-Tovar $^{2}$, \\ Sara Alicia Quiroz-Barroso², Blanca E. Buitrón-Sánchez ${ }^{3}$ \\ ${ }^{1}$ Posgrado en Ciencias Biológicas, Universidad Nacional Autónoma de México, Ciudad Universitaria, 04510 México, D.F., México \\ ${ }^{2}$ Museo de Paleontología, Facultad de Ciencias, Universidad Nacional Autónoma de México, Ciudad Universitaria, 04510 México, \\ D.F., México \\ ${ }^{3}$ Instituto de Geología, Universidad Nacional Autónoma de México, Ciudad Universitaria, 04510, México, D.F., México \\ *rvo17@yahoo.com.mx
}

\begin{abstract}
Resumen
Se reportan y describen once morfotipos de placas columnares de crinoides de edad misisípica y pensilvánica que han sido encontradas en diferentes niveles estratigráficos de las formaciones carboníferas Santiago (unidad informal) e Ixtaltepec, en sus afloramientos de la región de Santiago Ixtaltepec, Municipio de Nochixtlán, Oaxaca. Se propone a Axilinucrinus angustus como nuevo morfogénero y nueva morfoespecie. Cyclocaudex insaturatus y Floricyclus welleri se han encontrado en niveles del Misisípico medio (MeramecianoChesteriano?) del miembro superior de la formación Santiago. Lomalegnum cf. L. hormidium, Axilinucrinus angustus y un género indeterminado se presentan en niveles estratigráficos del Misisípico medio-tardío (Merameciano-Chesteriano) de la Formación Ixtaltepec. Pentagonomischus cf. P. plebeius y Cyclocion distinctus se encuentran en niveles del Pensilvánico temprano-medio (MorrowanoDesmoinesiano) de la Formación Ixtaltepec. Cylindrocauliscus fiski y Cyclocaudex typicus son morfotipos con una distribución muy amplia en la sección carbonífera de Nochixtlán. Existe una fuerte similitud entre todos los parataxa de Oaxaca con aquellos reportados del Misisípico inferior-Pensilvánico medio de la región del Mid-Continent en el centro-este de los Estados Unidos.
\end{abstract}

Palabras clave: Placas de crinoides, parataxonomía, Misisípico, Pensilvánico, Oaxaca, México.

\begin{abstract}
Eleven crinoid columnal morphotypes of Mississippian and Pennsylvanian ages found in different stratigraphic levels of the Santiago (informal unit) and Ixtaltepec formations in outcrops in the Santiago Ixtaltepec region, municipality of Nochixtlan, Oaxaca, are reported and described. Axilinucrinus angustus is proposed as a new morphogenus and new morphospecies. Cyclocaudex insaturatus and Floricyclus welleri have been found in middle Mississippian (Meramecian-Chesterian?) strata from the upper member of the Santiago formation. Lomalegnum $c f$. L. hormidium, Axilinucrinus angustus and one indeterminate genus are present in middle-late Mississippian (Meramecian-Chesterian) strata of the Ixtaltepec Formation. Pentagonomischus $c f$. P. plebeius and Cyclocion distinctus occur in earlymiddle Pennsylvanian (Morrowan-Desmoinesian) strata of the Ixtaltepec Formation. Cylindrocauliscus fiski and Cyclocaudex typicus are morphotypes with a broad distribution throughout the carboniferous section of Nochixtlan. A strong similarity exists between all of the paratax from Oaxaca and those reported from lower Mississippian-middle Pennsylvanian faunas of the Mid-Continent region of the central eastern United States.
\end{abstract}

Keywords: Crinoid plates, parataxonomy, Mississippian, Pennsylvanian, Oaxaca, Mexico. 


\section{Introducción}

De los invertebrados fósiles que se han descubierto en las rocas paleozoicas de la región de Santiago Ixtaltepec, municipio de Nochixtlán, Oaxaca, los crinoides carboníferos representan un componente abundante y diverso en las diferentes asociaciones que se han estudiado. La Clase Crinoidea está representada fundamentalmente por placas columnares conservadas generalmente como moldes en estratos de lutita y arenisca, en menor grado se presentan placas permineralizadas, ocasionalmente pluricolumnas y excepcionalmente cálices asociados a brazos y placas columnares. Dada la abundancia de las placas columnares y la importancia bioestratigráfica que este tipo de estructuras ha adquirido en la última década, en el presente estudio se describen los morfotipos que han sido encontrados hasta el presente en la región de Nochixtlán.

\section{Antecedentes}

El registro de crinoides del Paleozoico Superior de México es abundante, pero con la excepción del cáliz de la especie Parspaniocrinus beinerti del Pérmico de Coahuila (Strimple, 1971), el material referido en la literatura consiste principalmente de placas columnares disociadas que se han clasificado siguiendo la propuesta parataxonómica de Moore y Jeffords (1968). La presencia de placas se ha reportado para rocas del Paleozoico superior de los estados de Sonora, Coahuila, Tamaulipas, Hidalgo, Guerrero, Puebla y Chiapas (Buitrón-Sánchez, 1977; Villaseñor-Martínez et al., 1987; Velasco de León y Buitrón-Sánchez, 1992; Buitrón-Sánchez et al., 1987, 1998, 2007a, b; EsquivelMacías, 1996; Esquivel-Macías et al., 2000; Solís-Marín et al., 2004). Si bien una gran cantidad de placas aisladas pueden haber correspondido a un solo organismo, la abundancia y variedad de formas indica que el grupo fue un constituyente importante de las comunidades marinas del Paleozoico tardío.

En la región de Santiago Ixtaltepec, el material estudiado además de estar desarticulado, está pobremente preservado puesto que se han disuelto las placas dejando exclusivamente moldes, rasgo que había sesgado y dificultado anteriormente su estudio. Para solventar este problema, durante el desarrollo del trabajo se elaboraron, a partir de los moldes preservados principalmente en lutita, positivos en caucho-silicón que favorecen la observación y el estudio de la morfología de las placas disociadas.

\section{Localización del área de estudio y estratigrafía}

A $16 \mathrm{~km}$ al noreste de Nochixtlán, Oaxaca, entre los poblados de La Cumbre y Santiago Ixtaltepec (coordenadas $17^{\circ} 31^{\prime}$ y $17^{\circ} 33^{\prime}$ de latitud norte y $97^{\circ} 06^{\prime}$ y $97^{\circ} 07^{\prime}$ de longitud oeste), aflora una sucesión de rocas paleozoicas que consiste en depósitos marinos del Paleozoico temprano y tardío (Figura 1). Desde su descubrimiento en 1968 y posterior descripción (Pantoja-Alor, 1970), se han realizado estudios de los fósiles contenidos en ellas (Sour-Tovar y Quiroz-Barroso, 1991; Quiroz-Barroso y Perrilliat, 1997, 1998; Quiroz-Barroso et al., 2000; Navarro-Santillán et al., 2002; Landing et al., 2010; Streng et al., 2011, entre otros).

Las rocas más antiguas que afloran en el área corresponden al llamado Complejo Oaxaqueño de edad precámbrica, que está compuesto de gneiss, esquisto y pegmatita (Fries et al., 1962). Suprayaciendo a estas rocas se presenta una sucesión de roca calcárea y lutita con briozoarios, braquiópodos, gasterópodos, cefalópodos, trilobites y graptolitos de edad Cámbrico-Ordovícica, correspondiente a la Formación Tiñú (Figura 2).

Las rocas del Carbonífero están representadas por las formaciones Santiago (unidad informal por homonimia) e Ixtaltepec, que previamente fueron asignadas al Misisípico temprano (Osageano) y Pensilvánico temprano-medio (Morrowano-Desmoinesiano), respectivamente (PantojaAlor, 1970; Quiroz-Barroso y Perrilliat, 1997, 1998). Ambas unidades son portadoras de abundantes restos de esponjas, conuláridos, corales tabulados, briozoarios, braquiópodos,

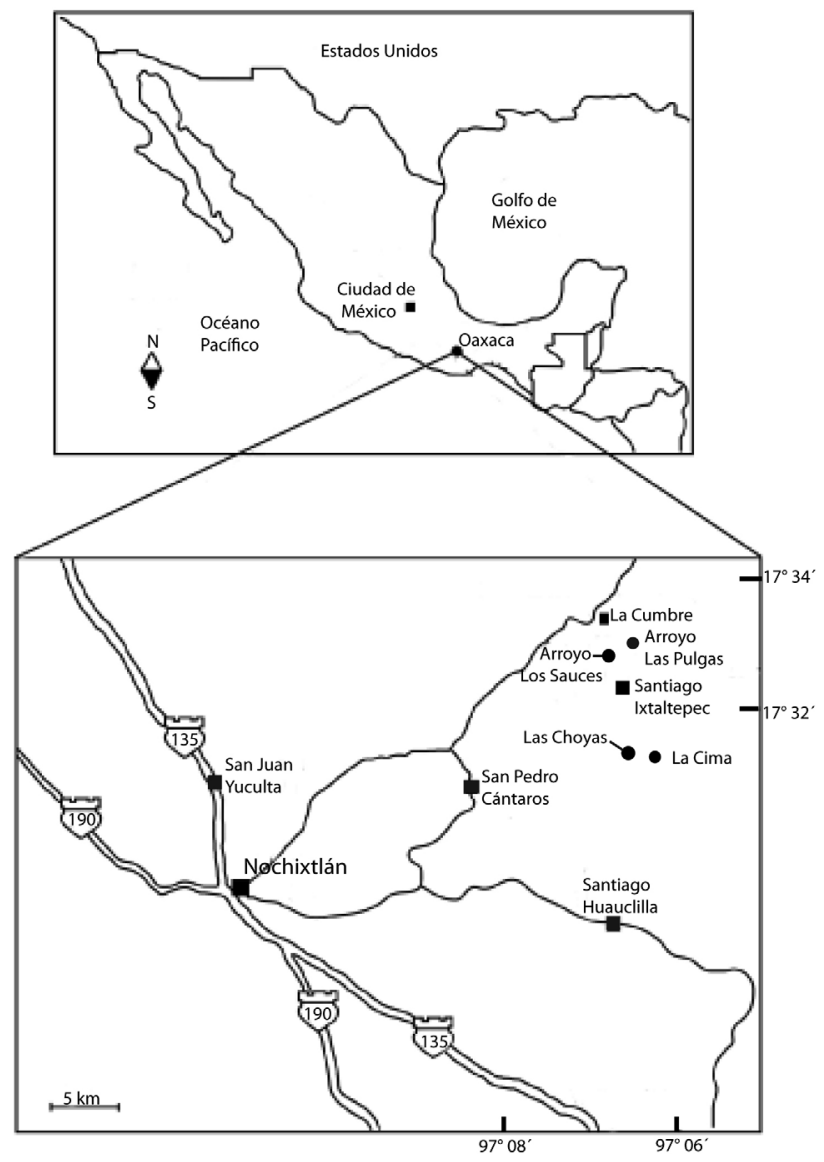

Figura 1. Ubicación geográfica del area de estudio, Santiago Ixtaltepec, Oaxaca. 


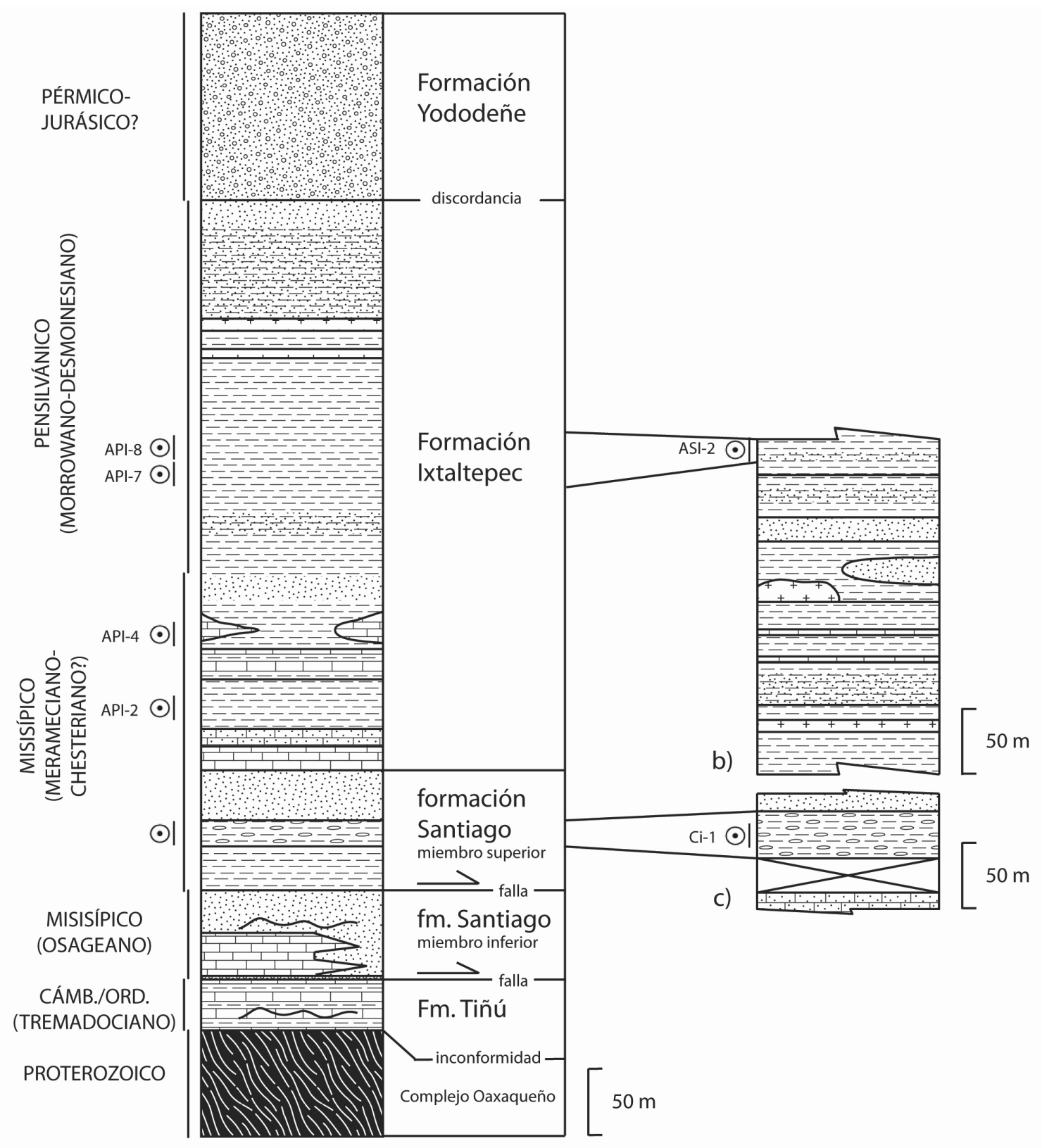

a)

\begin{tabular}{|c|c|c|c|c|c|}
\hline ro & Capas rojas & $\begin{array}{ll}1 \\
1\end{array}$ & Caliza & Nilll & Gneiss \\
\hline $\begin{array}{l}E== \\
E=1\end{array}$ & Lutita & 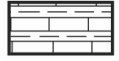 & Lutita calcárea & ${ }_{+}^{+}+{ }_{+}^{+}+$ & Roca intrusiva \\
\hline $\begin{array}{l}5=2 \\
=-2 \\
=-9=3\end{array}$ & Lutita con concreciones & $\begin{array}{ll}\cdots \\
\cdots\end{array}$ & Calcarenita & & gada y cizallada \\
\hline 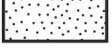 & Arenisca & 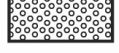 & Conglomerado matriz de caliza & $\Longrightarrow$ & Falla de bajo ángulo \\
\hline
\end{tabular}

Figura 2. Columnas estratigráficas de a) Arroyo de Las Pulgas (Modificado de Quiroz-Barroso y Perrilliat, 1998 y de Navarro-Santillán et al., 2002); b) Arroyo de Los Sauces (Modificado de Quiroz-Barroso y Perrilliat, 1998); c) La Cima. Los niveles de procedencia de los fósiles estudiados se señalan en el texto. 
gasterópodos, bivalvos, cefalópodos y crinoides, organismos que formaron parte de comunidades postarrecifales y arrecifales que se depositaron in situ o que en algunos casos fueron transportadas hacia zonas más profundas. De acuerdo a Pantoja-Alor (1970) la formación Santiago, en su sección tipo del Arroyo de las Pulgas, comprende 192 $\mathrm{m}$ de sedimentos de origen marino consistentes de caliza, arenisca, limolita y lutita que sobreyacen en discordancia angular a las rocas de la Formación Tiñú; con base en su litología, la dividió en dos miembros: el inferior calcáreo, que inicia con un conglomerado fino de cuarzo cementado por caliza amarilla ligeramente arcillosa; le sigue una caliza amarillenta con bandas de 5-20 cm de fragmentos finos de cuarzo y pasa a una caliza de color gris claro con abundantes braquiópodos, esponjas y corales, que en su parte superior cambia a caliza arenosa de estratificación delgada de color amarillo, con intercalaciones delgadas de lutita limolítica y marga con abundantes moldes de braquiópodos, bivalvos, tallos de crinoides e impresiones de briozoarios y corales. Quiroz-Barroso et al. (2000) reportaron para esta unidad el rostroconcho Pseudomulceodens que señala una edad osageana (Misisípico inferior) para las rocas portadoras, edad que Navarro-Santillán et al. (2002) confirman al describir para la misma unidad diversos braquiópodos y encontrar la asociación Lamellosathyris lamellosus Torynifer pseudolineatus, tipicamente osageana. En la misma sección tipo, el miembro superior de la formación Santiago es lutítico e inicia con una lutita en bancos gruesos, de colores abigarrados, seguida por arenisca calcárea amarillenta de grano fino con intercalaciones de capas delgadas de marga limolítica; hacia la parte media se presenta una arenisca poco calcárea y amarillenta que presenta cuarzo, feldespato y mica en una matriz arcillosa (subgrauvaca cuarzosa); la parte superior está formada por lutita abigarrada en bancos guesos con intercalaciones de arenisca calcárea que en algunas partes contienen conchas de braquiópodos, moldes de moluscos y tallos de crinoides muy mal preservados. En los últimos años se inició el estudio de afloramientos de este miembro en el cerro Inutoto y en sus alrededores, en donde se presenta una sucesión de rocas similar a la de la sección tipo del Arroyo de las Pulgas pero, en donde a diferencia de ésta, en diversos niveles de lutita abundan concreciones que llegan a contener diversas especies de conuláridos, braquiópodos, gasterópodos, bivalvos, nautiloideos, amonoideos, crinoideos y otros invertebrados marinos. Los estudios de esta fauna (CastilloEspinoza, 2008; Castillo-Espinoza et al., 2010; EscalanteRuiz, 2011) indican claramente una edad del Misisípico temprano-medio (Merameciano-Chesteriano).

Las rocas de la formación Santiago pasan transicionalmente a los clásticos suprayacentes de la Formación Ixtaltepec, que en el Arroyo de Las Pulgas tiene un espesor de cerca de $400 \mathrm{~m}$. Inicia con limolita, arenisca calcárea de grano fino y capas delgadas de calcarenita ligeramente arcillosa que pasan a bancos gruesos de lutita abigarrada ligeramente arenosa con intercalaciones de arenisca de grano fino; inmediatamente encima se encuentran una limolita y una arenisca de grano fino, micácea y finalmente bancos gruesos de lutita arenosa delimitados por estratos de arenisca de grano fino $\mathrm{y}$ limolita. La Formación Ixtaltepec presenta una abundante fauna representada por corales solitarios, briozoarios, braquiópodos (Sour-Tovar y Quiroz-Barroso, 1989; SourTovar y Martínez-Chacón, 2004; Torres-Martínez et al., 2008), gasterópodos, bivalvos (Quiroz-Barroso y Perrilliat, 1997, 1998), trilobites (Morón-Ríos y Perrilliat, 1988) crinoides y otros invertebrados, así como restos vegetales. De la fauna estudiada se ha logrado definir que los estratos de la parte media y superior de la Formación Ixtaltepec son de edad pensilvánica (Morrowano-Desmoinesiano) $\mathrm{y}$ estudios en proceso han detectado la presencia de braquiópodos, como Inflatia y Sinuatella, que indican que los estratos basales de la misma unidad son del Misisípico medio-superior (Merameciano-Chesteriano).

La secuencia paleozoica termina con el conglomerado de clastos de caliza con fusulínidos de la Formación Yododeñe, lo que indica una edad del Pérmico Tardío al Jurásico Temprano (Flores de Dios et al., 2000). Sobre esta formación se encuentran rocas calcáreas del Cretácico (Pantoja-Alor, 1970).

El material estudiado proviene de cuatro localidades (Figura 1). La primera de ellas es el Arroyo de Las Pulgas, en la que se han realizado recolectas en diferentes niveles estratigráficos de la Formación Ixtaltepec nominados como API-2 y API-4, del Merameciano-Chesteriano, y API-7 y API-8 del Morrowano-Desmoinesiano. La segunda localidad es el Arroyo de Los Sauces, en la que aflora la Formación Ixtaltepec y en la que el nivel ASI-2, se correlaciona con los niveles API-7 y API-8. La tercera localidad es La Cima, en donde aflora el miembro superior de la formación Santiago; en esta unidad el nivel Ci-1 es el único con abundantes fósiles del Merameciano. La cuarta localidad, llamada Las Choyas, está compuesta por un pequeño afloramiento que se correlaciona con los niveles API-2 y API-4 de la sección del Arroyo de las Pulgas de la Formación Ixtaltepec.

\section{Paleontología sistemática}

El material de estudio se encuentra depositado en las colecciones del Museo de Paleontología de la Facultad de Ciencias de la Universidad Nacional Autónoma de México (FCMP). Las placas columnares que se describen se clasifican de acuerdo a la propuesta parataxonómica de Moore y Jeffords (1968).

\subsection{Pentagonomischus plebeius}

Subclase y Orden inciertos Moore y Jeffords, 1968 Grupo Pentameri Moore y Jeffords, 1968

Familia Pentacauliscidae Moore y Jeffords, 1968 
Género Pentagonomischus Moore y Jeffords, 1968

Especie tipo Pentagonomischus plebeius Moore y Jeffords, 1968.

\subsubsection{Diagnosis}

Tallo pentagonal, heteromórfico; noditaxis de cuatro columnales; nodales cirríferos y no cirríferos, moderadamente más anchos y más altos que los priminternodales; lados de los columnales finamente granulosos; nodales e internodales más altos con una cresta angulosa finamente denticulada o con solo un borde redondeado; nodales cirríferos presentan de una a tres pequeñas cicatrices cirrales en la epifaceta; canal axial con un claustro delgado a la mitad de la altura de los columnales y bordeado por un yúgulo pequeño y estelado; lumen grande y circular; areola ancha y lisa, ligeramente cóncava, que se extiende hacia el borde del lumen; crenulario con crénulas cortas y fuertes dirigidas hacia el interior del margen.

\subsubsection{Descripción}

\section{Pentagonomischus cf. Pentagonomischus plebeius Moore y Jeffords, 1968 \\ Figura 3 (a-c)}

Perfil longitudinal de la placa redondeado; placa de forma pentagonal; faceta articular pentagonal; canal axial con claustro delgado; yúgulo de tamaño medio y estelado en el interior del lumen; lumen de contorno irregular, de un ancho ligeramente mayor al del yúgulo; areola lisa y subpentagonal; crenulario con crénulas cortas y gruesas; cúlmenes ligeramente más cortos que las crenelas.

Tallo asociado heteromórfico; noditaxis de ocho placas en la parte proximal, de 16 en la parte media y de 32 en la parte distal; nodales más anchos y más altos con respecto a los internodales; internodales de primer orden más anchos y altos que los de segundo orden; los de segundo orden, más anchos y altos que los de tercer orden, y así sucesivamente; lados de nodales muy redondeados y convexos, con epifacetas anchas y redondeadas, en algunos casos simulando una cresta poco conspicua a la mitad de la altura de los internodales; nodales sin cicatrices cirrales aparentes; suturas crenuladas.

\subsubsection{Discusión}

El material de Pentagonomischus cf. Pentagonomischus plebeius de la Formación Ixtaltepec se distingue de Asteromischus stellatus del Chesteriano de Illinois (Moore y Jeffords, 1968), la cual presenta placas de forma estelada, una epifaceta con un patrón pinado y el canal axial subpentagonal, más que estelado. Se diferencia de Pentaridica del Pensilvánico medio-superior de Estados Unidos (Moore y Jeffords, 1968), que tiene el canal axial generalmente circular, crénulas más largas, una areola de pentagonal a estelada, de cóncava a hundida hacia el lumen y una epifaceta más reducida o ausente. Difiere de Pentagonostaurus (Moore y Jeffords, 1968) que posee crénulas más gruesas y en menor número que la especie de Ixtaltepec y un lumen circular y sin yúgulo visible. Moore y Jeffords (1968) describen a Pentagonomischus plebeius? separado de P. plebeius, debido a que el primero aparece con un lumen fuertemente estelado y limitado por un aro circular. No obstante, los mismos autores indican que estos rasgos son diferencias menores del ejemplar tipo. El material oaxaqueño es comparable a $P$. plebeius? por lo que su determinación queda conferida a $P$. plebeius.

\subsubsection{Ocurrencia}

El material descrito fue encontrado en el Arroyo Las Pulgas (API-8), Formación Ixtaltepec y en el Arroyo de Los Sauces (ASI-2) de la misma formación. Ambos niveles se asignan al Pensilvánico (Morrowano-Desmoinesiano).

\subsubsection{Distribución}

Pentagonomischus plebeius se ha reportado para el Misisípico superior (Chesteriano) de Illinois (Moore y Jeffords, 1968). En México el género Pentagonomischus se ha encontrado en rocas del Misisípico inferior de San Salvador Patlanoaya, Puebla (Villaseñor-Martínez et al., 1987).

\subsubsection{Material descrito}

Ejemplares FCMP 762 (una placa), FCMP 763a, FCMP $763 b$ y FCMP 763c.

\subsection{Lomalegnum hormidium}

Grupo Cyclici Moore y Jeffords, 1968

Familia Cyclomischidae Moore y Jeffords, 1968

Género Lomalegnum Moore y Jeffords, 1968

Especie tipo: Lomalegnum hormidium Moore y Jeffords, 1968

\subsubsection{Diagnosis}

Tallo heteromórfico de apariencia homeomórfica, con columnales de alturas variables y cicatrices cirrales; lados de columnales rectos, granulosos y caracterizados especialmente por la apariencia bordada de las suturas, producto de un ligero alargamiento periférico de las terminaciones de los cúlmenes; suturas finamente crenuladas.

\subsubsection{Descripción}

\section{Lomalegnum cf. Lomalegnum hormidium}

Figura 3 (d-f)

Columna aparentemente homeomórfica en la que la presencia de nodales e internodales evidencia su carácter heteromórfico; noditaxis mínima de cuatro columnales; lados de columnales rectos y lisos; nodales ligeramente 

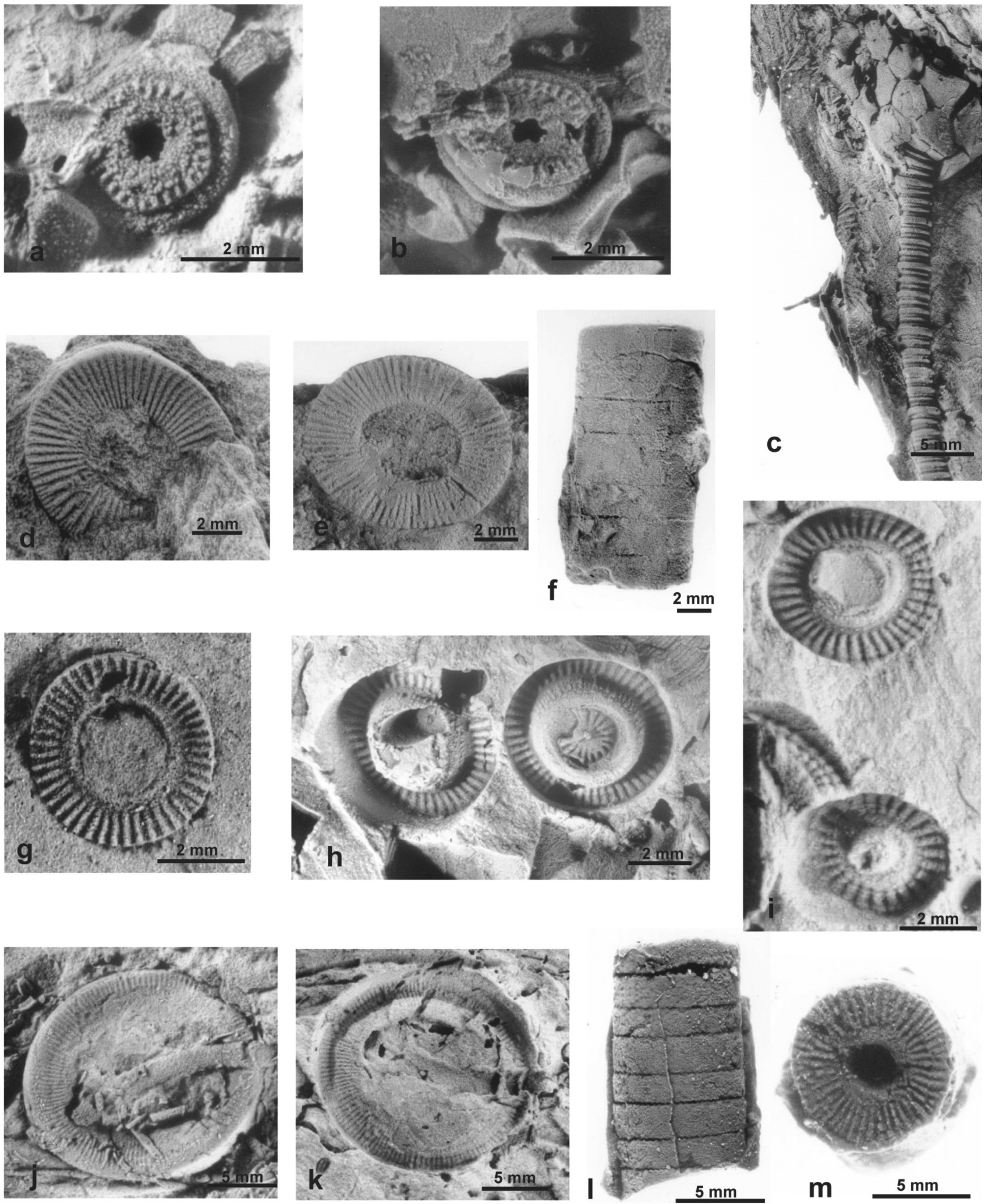

m

$5 \mathrm{~mm}$

Figura 3. (a-c) Pentagonomischus cf. Pentagonomischus plebeius. Arroyo los Sauces, Formación Ixtaltepec: a, ejemplar FCMP 763a; b, ejemplar FCMP 763b; c, ejemplar FCMP 763c. (d-f) Lomalegnum cf. Lomalegnum hormidium. Arroyo Las Pulgas, Formación Ixtaltepec: d, FCMP 770; e, ejemplar FCMP 769; f, FCMP 764a. (g-i) Axilinucrinus angustus gen. y sp. nov. Arroyo Las Pulgas, Formación Ixtaltepec: g, FCMP 772; h, FCMP 767d (izquierda) y FCMP 767e (derecha, holotipo); i, FCMP 767b (abajo), FCMP 767 (arriba). (j, k) ?Graphosterigma sp. Localidad Las Choyas, Formación Ixtaltepec: j y k, ejemplar FCMP 774 (molde y contramolde). $(1, \mathrm{~m})$ Cylindrocauliscus fiski. Localidad La Cima, miembro superior, formación Santiago: 1 y m, ejemplar FCMP 782. 
más altos que los internodales; nodales e internodales del mismo diámetro y nodales con dos cicatrices cirrales en polos opuestos; faceta articular circular con un lumen circular grande, rodeado de un crenulario amplio que abarca la mitad del tamaño de la faceta; crénulas delgadas, rectas, en su gran mayoría sin bifurcaciones y con crenelas de ancho similar al de los cúlmenes; en algunos ejemplares, el lumen aparenta estar rodeado por un perilumen muy delgado que lo separa del crenulario; suturas crenuladas. Medidas (en mm) e índices del ejemplar FCMP 767a.

Diámetro de la faceta $(\mathrm{F}), 5.36$; diámetro del lumen (L) 2.7 mmíndice diámetro del lumen/diámetro de la faceta $\mathrm{X}$ 100 (Li), 50.4; diámetro del crenulario (C), 2.66; índice diámetro del crenulario/diámetro de la faceta X $100(\mathrm{Ci})$, 49.6 .

\subsubsection{Discusión}

Se comparó el material de Lomalegnum cf. Lomalegnum hormidium de la región de Santiago Ixtaltepec con $L$. hormidium (Moore y Jeffords, 1968) de Iowa, EUA, que se diferencia porque la especie de Iowa presenta un perfil longitudinal granuloso, crénulas más delgadas y en su mayoría bifurcadas, el valor del índice Li ligeramente menor y el valor del índice Ci ligeramente mayor. También se comparó con Rhysocamax del Misisípico Inferior de Iowa (Moore y Jeffords, 1968); sin embargo, Rhysocamax tiene placas pequeñas de poca altura, lados de columnales redondeados longitudinalmente o con quillas, el índice $\mathrm{Li}$ de la especie tipo (R. cristata) es menor, mientras que el índice diámetro del crenulario/diámetro de la faceta $(\mathrm{Ci})$ en la misma especie es mayor. Debido a esto, el material de Oaxaca se confiere a $L$. hormidium por presentar un mayor número de características diagnósticas que con Rhysocamax.

\subsubsection{Ocurrencia}

Los ejemplares descritos fueron hallados en los niveles estratigráficos API-2 y API-4 (Misisípico, MeramecianoChesteriano) de la Formación Ixtaltepec en la sección del Arroyo de Las Pulgas.

\subsubsection{Distribución}

L. hormidium se ha reportado para el Osageano (Misisípico Inferior) de la Caliza Burlington, Iowa, EU (Moore y Jeffords, 1968) y para el Misisípico inferior de la Formación Patlanoaya de Puebla, México (VillaseñorMartínez et al., 1987; Esquivel-Macías, 1996).

\subsubsection{Material descrito}

Ejemplares FCMP 764a, FCMP 764b, FCMP 764c, FCMP 765a y FCMP 765b, FCMP 766a, FCMP 766b, FCMP 767a, FCMP 768, FCMP 769 y FCMP 770.

\subsection{Axilinucrinus}

Género Axilinucrinus nuevo género Villanueva-Olea y Sour-Tovar
Figura 3 (g-i)

\subsubsection{Diagnosis}

Cyclomischidae con un canal axial ornamentado con una serie de líneas, que lo recorren a lo largo y con un crenulario angosto.

\subsubsection{Descripción}

Ver descripción de Axilinucrinus angustus.

\subsubsection{Etimología.}

Del latín axis (eje) linus (línea) y crinus (crinoideo), que refiere al canal axial con una serie de líneas en sus paredes.

\subsubsection{Discusión}

El género identificado se ha colocado dentro de la familia Cyclomischidae por la presencia de un lumen circular grande y por carecer de una areola y perilumen. El crenulario en el ejemplar aquí descrito es angosto; por el contrario en los morfogéneros descritos previamente para la familia Cyclomischidae, es amplio. Lomalegnum del Misisípico inferior de Iowa (Moore y Jeffords, 1968) es el morfogénero más parecido al nuevo taxón pero el índice del lumen (Li) es menor y no presenta las características diagnósticas del nuevo morfogénero.

\subsubsection{Axilinucrinus angustus}

\section{Axilinucrinus angustus nueva especie Villanueva-Olea y Sour-Tovar}

Figura 3 (g-i)

\subsubsection{Diagnosis.}

Ver diagnosis de Axilinucrinus n. gen.

\subsubsection{Descripción}

Lados de los columnales rectos y lisos; faceta circular con un lumen circular grande, ocupando más de la mitad del diámetro de la faceta; crenulario delgado con crénulas rectas que en pocos ejemplares se bifurcan en la parte media del crenulario; cúlmenes tan anchos como las crenelas; paredes del canal axial con una serie de líneas que corren desde el borde del lumen hasta su interior. Medidas (en mm) e índices del ejemplar FCMP 767e: diámetro de la faceta (F), 4.9; diámetro del lumen (L), 3.39, índice diametro del lumen/diámetro de la faceta X $100(\mathrm{Li}), 68.5$; diámetro del crenulario $(\mathrm{C}), 1.56$, índice diámetro del crenulario/diámetro de la faceta X $100(\mathrm{Ci}), 31.5$.

\subsubsection{Etimología}

El nombre de la especie proviene del latín angustus (angosto o estrecho), que refiere al crenulario estrecho, característico del taxón.

\subsubsection{Tipos}

Holotipo FCMP 767e. Paratipos FCMP 767b, FCMP 
767c, FCMP 767d, FCMP 771, FCMP 772 y FCMP 773.

\subsubsection{Discusión}

En los niveles de la Formación Ixtaltepec en que se han recolectado, la abundancia de ejemplares, preservados en impresiones autígenicas, permite aseverar que la morfología de Axilinucrinus angustus no es una variación de otros taxa descritos previamente.

\subsubsection{Ocurrencia}

Los especímenes se recolectaron en capas de lutita de los niveles API-2 y API-4 (Misisípico, MeramecianoChesteriano) de la Formación Ixtaltepec en su sección del Arroyo Las Pulgas.

\subsubsection{Material descrito}

Además de los ejemplares tipo, en la colección del Museo de Paleontología de la Facultad de Ciencias de la UNAM, se encuentran depositados cerca de 50 especímenes de placas columnares de Axilinucrinus angustus.

\subsection{Graphosterigma}

Género Graphosterigma Moore y Jeffords, 1968

Especie tipo: Graphosterigma scriptum Moore y Jeffords, 1968

\subsubsection{Diagnosis}

Tallo heteromórfico, llegando a aparentar ser homeomórfico, moderadamente robusto; columnales casi idénticas en altura, con una ligera quilla, a manera de cresta arrugada o finamente tuberculada, ubicada a la mitad de la placa; faceta articular típica de la familia; crenulario ancho que rodea a un lumen grande y circular.

\subsubsection{Descripción}

\section{?Graphosterigma sp.}

Figura $3(\mathrm{j}, \mathrm{k})$

Perfil longitudinal de la placa convexo, con una quilla poco conspicua a la mitad de la placa; faceta articular de contorno circular, crenulario grande, con crénulas delgadas y rectas que se hunden hacia la luz del lumen; límite entre el lumen y el crenulario poco definido debido a la mala preservación, lumen con apariencia circular, muy amplio ocupando alrededor de tres cuartas partes del diámetro de la faceta; sin areola; suturas crenuladas. Medidas (en mm) e índices del ejemplar FCMP 774: diámetro de la placa (K), 15.18; diámetro de la faceta $(\mathrm{F}), 14.32$; índice diámetro de la faceta/diámetro de la placa X 100 (Fi), 94.3; diámetro de la epifaceta (E), 0.86; índice diámetro de la epifaceta/diámetro de la placa X 100 (Ei), 5.7; diámetro del lumen (L), 10.55; índice diámetro del lumen/diámetro de la faceta X 100 (Li), 73.7; diámetro del crenulario (C), 3.77; índice diámetro del crenulario/diámetro de la faceta X $100(\mathrm{Ci}), 26.3$.

\subsubsection{Discusión}

El crenulario es de un diámetro mayor, pero es visiblemente muy corto debido a que éste se hunde hacia el interior de la placa, posiblemente producto de una mala preservación. Se compararon las distintas placas de este morfotipo con el género Rhysocamax del Misisípico inferior de Iowa (Moore y Jeffords, 1968) que se distingue por sus columnales muy delgadas. El material de Ixtaltepec se distingue de Lomalegnum hormidium del Misisípico inferior de Iowa (Moore y Jeffords, 1968) que no tiene quillas en su perfil longitudinal y posee un lumen de menor tamaño. El material oaxaqueño se asigna con duda a Graphosterigma dada la condición hundida del crenulario, que no se presenta dentro de ninguna especie de este género, y que da la apariencia de un crenulario mucho muy reducido en diámetro.

\subsubsection{Ocurrencia}

El ejemplar descrito proviene de la localidad Las Choyas (Misisípico, Merameciano-Chesteriano), ladera oeste del Cerro de Inutoto, correspondiente a la base de la Formación Ixtaltepec.

\subsubsection{Distribución}

G. scriptum se ha referido para el Misisípico inferior de Kentucky (Moore y Jeffords, 1968); G. synthetes, para el Misisípico inferior (Osageano) de la Lutita New Providence, Grupo Borden en Kentucky (Moore y Jeffords, 1968); en tanto, G. grammodes se reporta para el Misisípico Inferior (Osageano) de la Formación Edwardsville, Grupo Borden en Indiana (Moore y Jeffords, 1968).

\subsubsection{Material descrito}

Ejemplar FCMP 774 (una placa que consta de molde y contramolde).

\subsection{Cylindrocauliscus}

Género Cylindrocauliscus Moore y Jeffords, 1968

Especie tipo: Cylindrocauliscus fiski Moore y Jeffords, 1968

\subsubsection{Diagnosis}

Tallo heteromórfico, de tamaño medio; noditaxis de 4 columnales; perfil longitudinal ligeramente convexo, faceta articular circular, lumen circular grande, rodeado por un delgado perilumen y un crenulario amplio; crénulas ligeramente gruesas, rectas, aunque algunas bifurcadas.

\subsubsection{Descripción}

Cylindrocauliscus fiski

Figura $3(1, m)$ y Figura 4 (a)

Lados de las columnales lisos y rectos a ligeramente convexos, algunos nodales presentan cicatriz cirral; faceta 

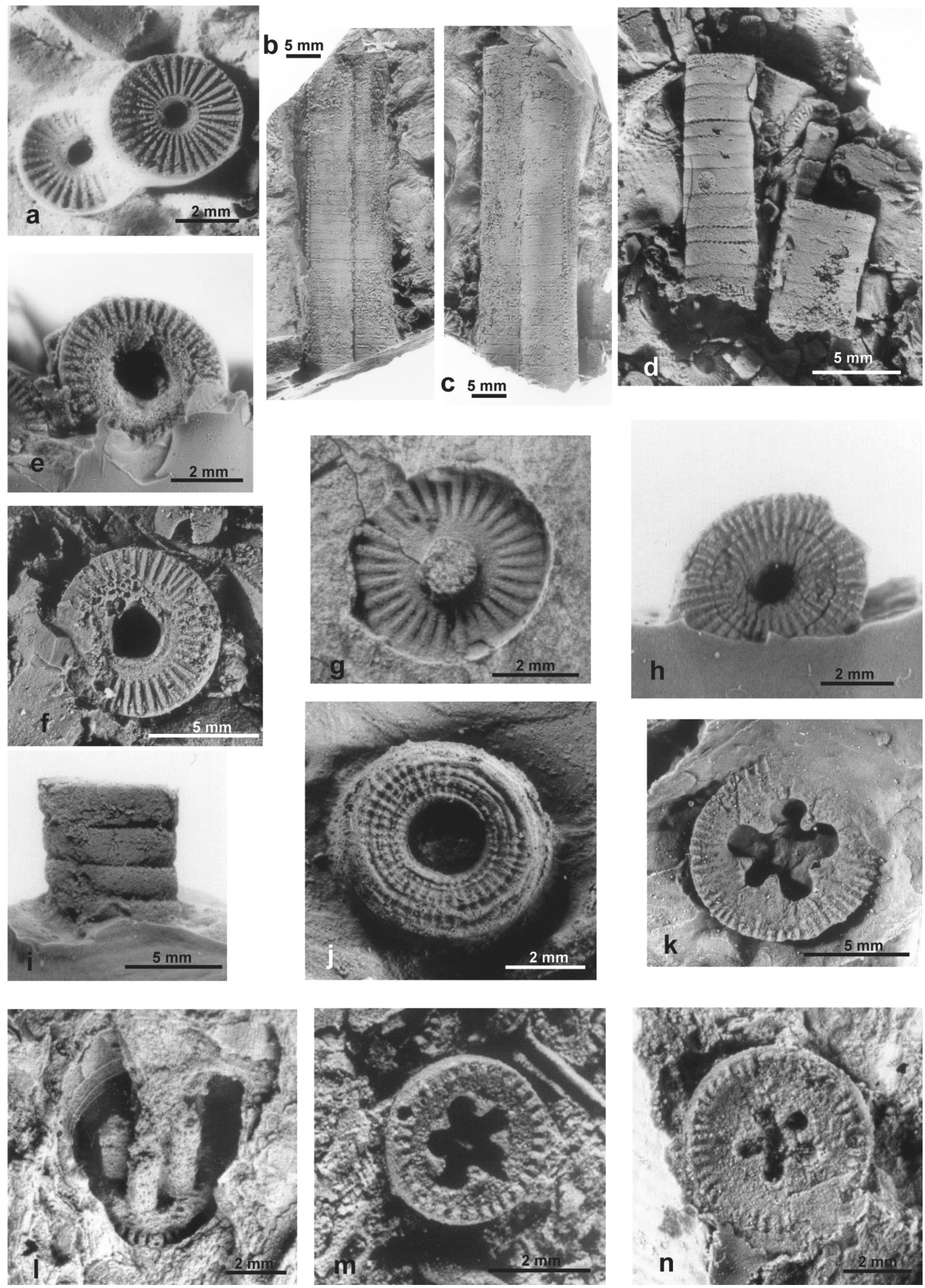

Figura 4. (a) Cylindrocauliscus fiski. Arroyo Las Pulgas, Formación Ixtaltepec: ejemplar FCMP 775. (b, c) Cyphostelechus cf. Cyphostelechus claudus. Las Choyas, Formación Ixtaltepec: b y c, ejemplar FCMP 785. (d, e, g) Cyclocaudex typicus. Arroyo Las Pulgas: d, ejemplares FCMP 789a (izquierda) y FCMP 789b (derecha); e, ejemplar FCMP 789a; g, FCMP 793. (f) Cyclocaudex typicus. Ejemplar de La Cima, miembro superior, formación Santiago: ejemplar FCMP 792. (h, i) Cyclocaudex insaturatus. Localidad La Cima, miembro superior, formación Santiago: h, i, ejemplar FCMP 795a. (j) Género indeterminado. Arroyo Las Pulgas: ejemplar FCMP 797. (k) Floricyclus welleri. La Cima, miembro superior, formación Santiago: ejemplar FCMP 795b. (1-n) Cyclocion distinctus. Arroyo Las Pulgas, Formación Ixtaltepec: 1, ejemplar FCMP 801; m, FCMP 790e; n, FCMP 799. 
articular circular; lumen circular de mediano a chico; perilumen angosto; crenulario de tamaño grande, con un promedio de 30 crénulas, rectas y gruesas, algunos cúlmenes son ligeramente más anchos que las crenelas. Suturas crenuladas. Medidas (en mm) e índices del ejemplar FCMP 775: diámetro de la faceta $(\mathrm{F}), 3.63$; diámetro del lumen (L), 0.88; índice diámetro del lumen/diámetro de la faceta X $100(\mathrm{Li}), 24.2$; diámetro del perilumen $(\mathrm{P}), 0.36$; índice diámetro del perilumen/diámetro de la faceta $(\mathrm{Pi}) \mathrm{X}$ 100, 9.9; diámetro del crenulario (C), 2.39; índice diámetro del crenulario/diámetro de la faceta X $100(\mathrm{Ci}), 65.9$.

\subsubsection{Discusión}

El morfotipo se diferencia de formas del género Heterostelechus del Pensilvánico superior - Pérmico Temprano de Texas (Moore y Jeffords, 1968), que tienen una areola cóncava y de mayor diámetro y presentan una progradación hacia un tracto elevado alrededor del lumen; difiere de Cycloscapus laevis del Pensilvánico medio de Texas (Moore y Jeffords, 1968) que posee una areola cóncava y en forma de tazón; de Preptopremnum del Pensilvánico medio-superior de Texas (Moore y Jeffords, 1968), que en su areola tiene irregularidades granulosas o vermiculadas; de Cyclocaudex insaturatus del Pensilvánico medio de Kansas (Moore y Jeffords, 1968), que presenta una noditaxis de 2 columnales y crénulas muy delgadas y casi todas bifurcadas. También fue comparado con Cyclocaudiculus regularis del Pensilvánico de Texas (Moore y Jeffords, 1968), del que se diferencia por que este último presenta un lumen más grande y areola rugosa. Se determina como Cylindrocauliscus fiski del Pensilvánico medio de Texas (Moore y Jeffords, 1968) por presentar las características principales del taxón, excepto que los ejemplares de Oaxaca se caracterizan por la presencia de crénulas rectas, los lados de los columnales rectos y el lumen es más bien de tamaño medio a chico.

\subsubsection{Ocurrencia}

Los ejemplares analizados provienen del miembro superior de la formación Santiago, de la localidad La Cima (Misisípico, Merameciano); de los niveles API-2 y API-4 (Misisípico, Merameciano-Chesteriano) y API-7 y API-8 (Pensilvánico, Morrowano-Desmoinesiano) de la sección del Arroyo Las Pulgas de la Formación Ixtaltepec; y del nivel ASI-2 de la Formación Ixtaltepec en la sección del Arroyo Los Sauces (Pensilvánico, Morrowano-Desmoinesiano).

\subsubsection{Distribución}

C. fiski se ha reportado en la Lutita Mingus, Grupo Strawn, del Desmoinesiano (Pensilvánico medio) de Texas (Moore y Jeffords, 1968). Además, se ha reportado su presencia en los estados de Kansas, Ohio y Colorado (Simon y Geizer, 1976; Buitrón-Sánchez et al., 2008). En México, C. fiski se reporta para la Formación Del Monte en Calnali, Hidalgo (Buitrón-Sánchez et al., 1987), en la Formación Del Monte en el Cañón de La Peregrina, Tamaulipas (Buitrón-
Sánchez et al., 1998), en Pemuxco, Hidalgo (López-Lara, 2002; Buitrón-Sánchez et al., 2008) y en Cerros Las Mesteñas, Sonora (Buitrón-Sánchez et al., 2008). BuitrónSánchez asignó con cierta duda los crinoides encontrados en la Formación Santa Rosa Inferior, en Chiapas (BuitrónSánchez, 1977) a esta especie.

\subsubsection{Material descrito}

Ejemplares FCMP 775, FCMP 776, FCMP 777, FCMP 778, FCMP 779, FCMP 780, FCMP 781, FCMP 782, FCMP 783 y FCMP 784.

\subsection{Cyphostelechus}

\section{Género Cyphostelechus Moore y Jeffords, 1968}

Especie tipo: Cyphostelechus claudus Moore y Jeffords, 1968

\subsubsection{Diagnosis}

Tallo heteromórfico pero en apariencia homeomórfico; noditaxis aparente de cuatro columnales; costados columnales ligeramente convexos y de superficie lisa; nodales sin cirros en su perfil longitudinal; faceta articular con un lumen grande y circular; crenulario en su parte externa con crénulas rectas, igualmente dispuestas en el plano de la faceta, en su parte interna con continuación de las crénulas curvándose abruptamente hacia abajo desde el plano facetal hacia dentro del lumen; suturas de finamente crenuladas a indentadas.

\subsubsection{Descripción}

\section{Cyphostelechus cf. Cyphostelechus claudus Figura $4(b, c)$}

Tallo en apariencia homeomórfico en el que ligeras variaciones en la altura de las placas indica su carácter heteromórfico; columnales bajas, sin cirros; lados de columnales lisos; no hay conservación de la faceta articular completa, pero una pequeña fracción indica crénulas delgadas, en su mayoría rectas y excepcionalmente bifurcadas; cúlmenes igual de anchos que las crenelas; suturas de indentadas a ligeramente crenuladas.

\subsubsection{Discusión}

El tallo presenta un surco que corre a lo largo de su parte media y atraviesa toda su longitud. De acuerdo a la discusión de la especie original, esto es una condición que se repite en varios ejemplares por el colapso del tallo debido al gran tamaño del canal axial. La no conservación de una faceta completa no permite la asignación con certeza del ejemplar de Ixtaltepec a Cyphostelechus claudus del Desmoinesiano de Texas (Moore y Jeffords, 1968), pero la condición colapsada es evidente en el perfil longitudinal del tallo; esta condición sólo se ha reportado en este género 
monoespecífico.

\subsubsection{Ocurrencia}

Los ejemplares provienen de los niveles API-7 y API-8 (Pensilvánico, Morrowano-Desmoinesiano) de la Formación Ixtaltepec en su sección del Arroyo de las Pulgas.

\subsubsection{Distribución}

Cyphostelechus claudus sólo se ha reportado para el Pensilvánico medio (Desmoinesiano) de la Lutita Mingus, Grupo Strawn en Texas (Moore y Jeffords, 1968).

\subsubsection{Material descrito \\ Ejemplares FCMP 785, FCMP 786, FCMP 787 y FCMP 788.}

\subsection{Cyclocaudex}

Género Cyclocaudex Moore y Jeffords, 1968

Especie tipo: Cyclocaudex typicus Moore y Jeffords, 1968.

\subsubsection{Diagnosis}

Tallo heteromórfico; lados rectos a ligeramente convexos; nodales comúnmente con cicatrices cirrales, no más anchos que las internodales pero ligeramente más altos; faceta articular con un lumen pequeño a grande y de circular a subpentagonal; areola con un índice aproximadamente igual; crenulario amplio con crénulas rectas y largas.

\subsubsection{Descripción}

\section{Cyclocaudex typicus Moore y Jeffords, 1968}

Figura 4 (d, e, f, g)

Perfil longitudinal de recto a convexo, tallo heteromórfico con internodales hasta de tercer orden; nodales con cicatrices cirrales e igual de anchos que los internodales; faceta articular circular; lumen de tamaño medio a moderadamente grande, de forma circular a pentagonal; areola estrecha, lisa y en algunos casos ligeramente granulada; crenulario ancho, con crénulas de tamaño medio, gruesas, rectas y en ocasiones bifurcadas; cúlmenes igual de anchos que las crenelas; suturas crenuladas. Medidas (en $\mathrm{mm}$ ) e índices del ejemplar FCMP 792: diámetro de la faceta (F), 7.61; diámetro del lumen (L), 2.82; índice diámetro del lumen/ diámetro de la faceta X $100(\mathrm{Li}), 37$; diámetro de la areola (A), 1.15; índice diámetro de la areola/diámetro de la faceta X 100 (Ai), 15.1; diámetro del crenulario (C), 3.64; índice diámetro del crenulario/diámetro de la faceta X $100(\mathrm{Ci})$, 47.8 .

\subsubsection{Discusión}

Cyclocaudex plenus del grupo Cisco del Pensilvánico Superior de Texas (Moore y Jeffords, 1968), se diferencia de
C. typicus por que en el primero se observa un lumen muy pequeño y suturas no crenuladas. Cyclocaudex insaturatus de la Formación Cabaniss del Pensilvánico Medio de Kansas (Moore y Jeffords, 1968) se distingue del material estudiado, principalmente por carecer de areola, presentar un mayor número de crénulas, cúlmenes más largos y un solo internodal en su noditaxis. Cyclocaudex jucundus del Pensilvánico Superior de Texas (Moore y Jeffords, 1968), a diferencia de C. typicus, tiene una areola más ancha y deprimida. La especie Cyclocaudex costatus de la Formación Graham del Pensilvánico de Texas (Moore y Jeffords, 1968) posee un lumen bordeado de un perilumen y un menor número de crénulas.

\subsubsection{Ocurrencia}

Miembro superior de la formación Santiago en la localidad de la Cima, (Misisípico, Merameciano); niveles API-2 y API-4 (Misisípico, Merameciano-Chesteriano) y API-7 y API-8 (Pensilvánico, Morrowano-Desmoinesiano) de la Formacion Ixtaltepec en su sección del Arroyo de las Pulgas; nivel ASI-2 (Pensilvánico, MorrowanoDesmoinesiano) de la Formación Ixtaltepec en su sección del Arroyo de Los Sauces.

\subsubsection{Distribución}

El género Cyclocaudex ha sido reportado para la Formación Minturn del Pensilvánico Medio, en Colorado (Webster y Houck, 1998). Moore y Jeffords (1968) describen y nombran a varias especies de este género para el Pensilvánico Superior de Texas. En México el género Cyclocaudex ha sido encontrado en el Pensilvánico de Hidalgo (Buitrón-Sánchez et al., 1987) y Sonora (BuitrónSánchez et al., 2007a y b).

La especie Cyclocaudex typicus se ha registrado para la Caliza Belknap del Pensilvánico Superior de Texas (Moore y Jeffords, 1968), también para la Formación Patlanoaya del Pensilvánico Superior de Puebla, México (VillaseñorMartínez et al., 1987; Esquivel-Macías, 1996) y para el Wordiano temprano - medio de la Formación La Cruz en Ihualtepec, Oaxaca (Vachard et al., 1997).

\subsubsection{Material descrito}

Ejemplares FCMP 789a, FCMP 789b, FCMP 789c, FCMP 789d, FCMP 789e, FCMP 790a, FCMP 790b, FCMP 790c, FCMP 791, FCMP 792, FCMP 793 y FCMP 794.

\subsection{Cyclocaudex insaturatus}

\section{Cyclocaudex insaturatus Moore y Jeffords, 1968 Figura $4(h, i)$}

\subsubsection{Diagnosis}

Tallos pequeños y lumen circular grande, carente de areola; crénulas moderadamente finas y rectas (Moore y Jeffords, 1968). 


\subsubsection{Descripción}

Perfil longitudinal convexo; algunos nodales presentan cicatrices cirrales; faceta articular de circular a ligeramente ovalada, con diámetro de tamaño medio; el lumen es de tamaño medio, circular y carente de areola; el crenulario es amplio con un promedio de 37 crénulas; los cúlmenes son finos y rectos, algunos bifurcados en la proximidad del contorno de la placa; las crenelas son más anchas que los cúlmenes; suturas crenuladas.

\subsubsection{Discusión}

Aun cuando en la diagnosis elaborada por Moore y Jeffords (1968) se menciona que la especie Cyclocaudex insaturatus el lumen es grande, el material ilustrado por los mismos autores presenta lúmenes de tamaño medio a grande y en las mismas proporciones que poseen los ejemplares oaxaqueños. Cyclocaudex medius del Tournasiano de Polonia (Gluchowski y Racki, 2005) se distingue del material de la formación Santiago por presentar un lumen muy pequeño y de forma de circular a subpentagonal y una areola muy angosta. Cyclocaudex jucundus del Pensilvánico Superior de Texas (Moore y Jeffords, 1968), es diferente a los ejemplares estudiados por presentar una areola amplia, un perilumen prominente y un crenulario formado por cúlmenes y crenelas angostas.

El material de la formación Santiago también fue comparado con el morfotipo Cyclocaudex aptus del Borden Group del Misisípico de Kentucky (Moore y Jeffords, 1968), del que se diferencia por que este último posee una faceta articular con crénulas muy finas y carece de suturas crenuladas. Cyclocaudex paranaensis del Devónico de Brasil (Scheffler y Sequeira, 2007) es distinta de $C$. insaturatus ya que presenta un lumen pequeño y una areola muy amplia y deprimida, y crénulas simples.

\subsubsection{Ocurrencia}

La Cima (Ci-1), miembro superior de la formación Santiago (Misisípico, Merameciano).

\subsubsection{Distribución}

Cyclocaudex insaturatus se ha descrito para el Pensilvánico Medio de Kansas (Moore y Jeffords, 1968). En México ha sido registrada para el Pensilvánico de Sierra Agua Verde, Cerros Las Mesteñas y Cerros El Tule en Sonora, la Formación Del Monte en Calnali, Hidalgo, San Salvador Patlanoaya, Puebla y para la Formación Ixtaltepec del Pensilvánico de Oaxaca (Buitrón-Sánchez et al., 2008).

\subsubsection{Material descrito}

Dos placas inarticuladas, ejemplares: FCMP 795a y FCMP 796.

\subsection{Género indeterminado}

Género indeterminado
Figura $4(j)$

\subsubsection{Descripción}

Perfil longitudinal liso y recto; faceta circular; lumen circular de tamaño mediano rodeado de un crenulario ancho con crenelas ligeramente más anchas que los cúlmenes; sobre el crenulario corren líneas circulares, paralelas y siguiendo el contorno del lumen. No hay areola ni perilumen y las crénulas se deprimen hacia el contorno del lumen.

Medidas (en mm) e índices del ejemplar FCMP 797: diámetro de la faceta $(\mathrm{F}), 5.27$; diámetro del lumen $(\mathrm{L}), 2.01$; índice diámetro del lumen/diámetro de la faceta X 100 (Li), 38.1; diámetro del crenulario $(\mathrm{C}), 3.26$; índice diámetro del crenulario/diámetro de la faceta X $100(\mathrm{Ci}), 61.9$.

\subsubsection{Discusión}

La descripción se basa en un solo ejemplar que se asigna a la familia Cyclomischidae por la ocurrencia de un lumen circular con un crenulario ancho y por carecer de una areola y perilumen. Por lo mismo, se lo relaciona a Lomalegnum del Misisípico inferior de Iowa, Estados Unidos (Moore y Jeffords, 1968) y del Misisípico Inferior de Puebla, México (Villaseñor-Martínez et al., 1987; Esquivel-Macías, 1996), pero el hecho de poseer las líneas descritas sobre el crenulario no permite asegurar su asignación a este género. También presenta similitud con Axilinucrinus, descrito en este trabajo, pero se diferencia en que este morfotipo presenta un lumen más pequeño y en Axilinucrinus no se observa la ornamentación en el crenulario que caracteriza a este ejemplar.

\subsubsection{Ocurrencia}

El ejemplar proviene del nivel API-4 del Arroyo Las Pulgas, Formación Ixtaltepec (Misisípico, MeramecianoChesteriano).

\subsubsection{Material descrito Ejemplar FCMP 797.}

\subsection{Floricyclus welleri}

Familia Floricyclidae Moore y Jeffords, 1968

Género Floricyclus Moore y Jeffords, 1968

Especie tipo: Floricyclus hebes Moore y Jeffords, 1968

Floricyclus welleri Moore y Jeffords, 1968

Figura $4(\mathrm{k})$

\subsubsection{Diagnosis}

Lumen grande de forma petaloide con las extremidades de los rayos redondeados, areola lisa a ligeramente granulada, crénulas gruesas. (Moore y Jeffords, 1968).

\subsubsection{Descripción}

Perfil longitudinal convexo y liso; placas columnares 
de forma circular; lumen pentalobulado de tamaño medio, la parte exterior de los rayos es redondeada; perilumen ligeramente elevado; areola granulada y estrecha. Crenulario amplio compuesto por 53 crénulas en promedio; cúlmenes rectos, de tamaño grande, gruesos y en algunos casos bifurcados, del mismo grosor que las crenulas. Medidas (en mm) e índices del ejemplar FCMP 795b: diámetro de la faceta $(\mathrm{F}), 9.23$; diámetro del lumen (L), 4.14; índice diámetro del lumen/diámetro de la faceta X 100 (Li), 44.9; diámetro de la areola (A), 1.32; índice diámetro de la areola/diámetro de la faceta X 100 (Ai), 14.3; diámetro del crenulario $(\mathrm{C}), 3.77$; índice diámetro del crenulario/diámetro de la faceta X 100 (Ci), 40.8 .

\subsubsection{Discusión}

El ejemplar de la morfoespecie Floricyclus welleri procedente de la formación Santiago, tiene características similares a Cyclocion distinctus de la Formación Paint Creek del Misisípico de Illinois (Moore y Jeffords, 1968), tales como el lumen de tamaño medio de pentalobulado a floriforme y perilumen elevado, sin embargo, el ejemplar estudiado de Floricyclus welleri posee crénulas mas largas y una areola mas angosta. Lamprosterigma erathense del Pensilvánico medio de Texas (Moore y Jeffords, 1968), también presenta lumen pentalobulado, pero difiere de la morfoespecie de Oaxaca por su mayor tamaño y por poseer un perilumen circular que no sigue el contorno petaloide del lumen; L. erathense también se distingue por su faceta articular más amplia. Floricyclus welleri de la formación Santiago también se distingue de otras especies del género, como F. granulosus de la Formación Thrifty del Pensilvánico superior de Texas (Moore y Jeffords, 1968) que presenta una areola lisa y crénulas cortas y gruesas; y de $F$. hebes que, a diferencia de $F$. welleri, presenta un crenulario angosto y deprimido y una areola muy lisa.

\subsubsection{Ocurrencia}

Localidad La Cima, miembro superior de la formación Santiago (Misisípico, Merameciano).

\subsubsection{Distribución}

Floricyclus welleri ha sido descrita para la Formación Brodhead del Borden Group del Osageano de Kentucky (Moore y Jeffords, 1968) y para el Misisípico y Pensilvánico de la Formación Patlanoaya en Puebla (Esquivel-Macías et al., 2000; Villaseñor-Martínez et al., 1987).

\subsubsection{Material descrito}

Dos placas desarticuladas: FCMP 795b y FCMP 798.

\subsection{Cyclocion distinctus}

Género Cyclocion Moore y Jeffords, 1968

Especie tipo: Cyclocion distinctus Moore y Jeffords, 1968

\subsubsection{Diagnosis}

Lados de columnales levemente convexos, de lisos a granulosos, con tendencia a desarrollar una cresta apenas visible a la mitad de la altura de las columnales en perfil longitudinal; facetas articulares con lumen estelado grande, areola lisa y ancha, crenulario muy delgado; suturas crenuladas.

Cyclocion distinctus Moore y Jeffords, 1968

Figura $4(1, m, n)$

\subsubsection{Descripción}

Lados de las columnales lisos con perfil recto a convexo; nodales con cicatriz cirral y más altos que los internodales; internodales de primer orden más altos que los internodales de segundo orden; faceta articular circular; lumen grande que ocupa más de la mitad del diámetro total de la faceta, floriforme, rodeado por una areola lisa de gran tamaño; crenulario muy reducido en diámetro, con crénulas cortas, gruesas y rectas y crenelas de ancho similar al de los cúlmenes; suturas crenuladas. Medidas (en mm) e índices del ejemplar FCMP 790d: diámetro de la faceta (F), 4.73; diámetro del lumen (L), 2.08; índice diámetro del lumen/ diámetro de la faceta X $100(\mathrm{Li}), 44$; diámetro de la areola (A), 1.81; índice diámetro de la areola/diámetro de la faceta X 100 (Ai), 38.2; diámetro del crenulario (C), 0.84; índice diámetro del crenulario/diámetro de la faceta X $100(\mathrm{Ci})$, 17.8 .

\subsubsection{Discusión}

Los ejemplares de Oaxaca asignados a Cyclocion distinctus se distinguen de la forma tipo descrita para Illinois, Estados Unidos (Moore y Jeffords, 1968) por carecer de un perilumen visible; se considera que esta diferencia no es suficiente para hacer una distinción específica. C. distinctus se separa de Floricyclus del Misisípico inferior - Pensilvánico superior de Estados Unidos (Moore y Jeffords, 1968), que presenta un lumen con puntos delgados entre los rayos petaloides y una areola delgada. C. distinctus se diferencia de Cyclocrista del Pensilvánico superior - Pérmico inferior de Texas (Moore y Jeffords, 1968), que presenta el crenulario separado de la areola por un declive, la areola algo cóncava o levantada semejando un perilumen amplio y por el lumen que puede ser de circular a quinquelobulado.

\subsubsection{Ocurrencia}

Nivel API-7 del Arroyo Las Pulgas y nivel ASI-2 del Arroyo Los Sauces, Formación Ixtaltepec (Pensilvánico, Morrowano-Desmoinesiano).

\subsubsection{Distribución}

Cyclocion sp. se ha reportado para el Fameniano tardío de Kazakhstan Central (Sisova, 1988) y para el Fameniano de las Montañas Holy Cross en Polonia (Gluchowski, 2002). C. distinctus se ha encontrado en capas del Chesteriano (Misisípico superior) de la Formación Paint Creek en Illinois 
(Moore y Jeffords, 1968).

\subsubsection{Material descrito}

Ejemplares FCMP 790d, FCMP 790e, FCMP 799, FCMP 800 y FCMP 801.

\section{Discusión y conclusiones}

Las diferentes placas columnares que se describen representan en su mayoría parataxones ya referidos en otras localidades del Paleozoico superior de México; todos ellos, a excepción del nuevo morfogénero que se postula, han sido reportados para localidades misisípicas o pensilvánicas de la región centro-este de los Estados Unidos, conocida como Mid-Continent. Esta afinidad es coherente con las que presentan otros grupos de invertebrados de las formaciones Santiago e Ixtaltepec, principalmente braquiópodos y moluscos, que han sido estudiados previamente (QuirozBarroso y Perrilliat, 1997, 1998; Quiroz-Barroso et al., 2000; Navarro-Santillán et al., 2002; Sour Tovar y MartínezChacón, 2004; Torres-Martínez et al., 2008). Tal afinidad apoya las inferencias que se han hecho y que señalan que, durante el Carbonífero, la región sureste del territorio de México representó la extensión hacia el sur-suroeste del mar epicontinental que cubrió lo que actualmente es la región del Mid-Continent. Desde el punto de vista tectónico, esta información implica que a inicios del Carbonífero la porción litosférica que compone al este-sureste de México y que es conocida como el microcontinente de Oaxaquia (OrtegaGutiérrez et al., 1995) ya se encontraba en el margen sureste de la Placa Norteamericana.

En las secuencias misisípicas y pensilvánicas de la región de Nochixtlán, los tipos de placas columnares presentan una distribución estratigráfica particular y su asociación con géneros o especies de braquiópodos o cefalópodos considerados como índices permite establecer la edad en que se depositaron los especímenes estudiados. De los morfos estudiados, Cyclocaudex insaturatus y Floricyclus welleri se encuentran en niveles de la formación Santiago cuya edad se asigna al Misisípico medio (Merameciano) por la presencia de los cefalópodos Beyrichoceras hornerae, Goniatites crenistria y Prolecanites americanus. Los ejemplares de Lomalegnum cf. L. hormidium, Axilinucrinus angustus $\mathrm{n}$. gen. y sp. y el género indeterminado recolectados en los niveles de la base de la Formación Ixtaltepec (API-2 - API4) se encuentran asociados a especies de braquiópodos de los géneros Inflatia y Sinuatella que indican una edad de Misisípico medio-tardío (Merameciano-Chesteriano). Los crinoides Pentagonomischus cf. P. plebeius y Cyclocion distinctus encontrados en los niveles API-7 y API8 del Arroyo de Las Pulgas y ASI-2 se encuentran asociados a los braquiópodos Neochonetes granulifer y Neospirifer dunbari que son de edades pensilvánicas (Morrowano-Desmoinesiano), esta información establece la posibilidad de ampliar el alcance estratigráfico de ambos parataxones de crinoideos que previamente solo se habían reportado para edades misisípicas. Cylindrocauliscus fiski y Cyclocaudex typicus, formas previamente referidas para el Pensilvánico de Norteamérica, en los afloramientos de la región de Nochixtlán tienen una distribución muy amplia encontrándose tanto en estratos misisípicos como pensilvánicos por lo que también se amplían los alcances estratigráficos previamente reportados por Moore y Jeffords (1968).

\section{Agradecimientos}

Los autores agradecen a Daniel Navarro S. su colaboración durante el trabajo de campo y curatorial, a Leonora Martín M. por su asistencia técnica en el campo y por la elaboración de los moldes y duplicados en silicón, y a Héctor Hernández por la elaboración del material fotográfico que ilustra esta publicación. También se agradece a Carlos Esquivel Macías y Forest J. Gahn la revisión del trabajo, sus observaciones y sugerencias, que repercutieron en una mejora sustancial del manuscrito original. Este trabajo ha sido financiado por la Dirección General de Asuntos del Personal Académico de la UNAM a través de los proyectos PAPITT 209308 e IN213710.

\section{Referencias}

Buitrón-Sánchez, B.E., 1977, Invertebrados (Crinoidea y Bivalvia) del Pensilvánico de Chiapas: Revista del Instituto de Geología, 1, 144-150.

Buitrón-Sánchez, B.E., Patiño, J., Moreno, A., 1987, Crinoides del Paleozoico Tardío de Calnali, Hidalgo: Revista de la Sociedad Mexicana de Paleontología, 1, 125-136.

Buitrón-Sánchez, B.E., Arellano-Gil, J., Flores de Dios, L.A., 1998, Crinoides del Pensilvánico del Cañón de la Peregrina, Estado de Tamaulipas, México (resumen), en Primera Reunión Nacional de Ciencias de la Tierra: México D.F., México, Unión Geofísica Mexicana, 55.

Buitrón-Sánchez, B.E., Gómez-Espinosa C., Almazán-Vázquez, E., Vachard, D., 2007a, A late Atokan regional encrinite (early late Moscovian, Middle Pennsylvanian) in the Sierra Agua Verde, Sonora state, NW Mexico, en Álvaro, J.J., Aretz, M., Boulvain, F., Munnecke, A., Vachard, D., Vennin, E. (eds.), Paleozoic reefs and bioaccumulations: climatic and evolutionary controls: Geological Society Special Publications, 275, 201-209.

Buitrón-Sánchez, B.E., Almazán-Vázquez, E., Vachard, D., 2007b, Middle Permian crinoids (Echinodermata, Crinoidea) from Cerros Los Monos, Caborca, Sonora, Mexico and paleogeographic considerations: Revista Mexicana de Ciencias Geológicas, 24, 344-353.

Buitrón-Sánchez, B.E., Gómez-Espinosa, C., Almazán-Vázquez E., Vachard D., Laguarda-Figueras, A., Solís-Marín, F., 2008, A review of the crinoid columnals (Echinodermata-Crinoidea) from the Carboniferous of Mexico: Revista de Biología Tropical, 56, 1-12.

Castillo-Espinoza, K.M., 2008, Cefalópodos de la Formación Santiago, Misisípico de la Región de Nochixtlán, Oaxaca: México, D.F., Universidad Nacional Autónoma de México, tesis de licenciatura, $52 \mathrm{p}$. 
Castillo-Espinoza, K.M., Escalante-Ruiz, A.R., Quiroz-Barroso, S.A., Sour-Tovar, F., Navarro-Santillán, D., 2010, Nuevos invertebrados del Viseano (Mississippiano), Formación Santiago, Oaxaca, sudeste de México (resumen), en X Congreso Argentino de Paleontología y Bioestratigrafía y VII Congreso Latinoamericano de Paleontología: La Plata, Argentina, Asociación Geológica Argentina, 145.

Escalante Ruiz, A.R., 2011, Conuláridos y Gasterópodos del Carbonífero de Nochixtlán, Oaxaca: México, D.F., Universidad Nacional Autónoma de México, tesis de maestría, $86 \mathrm{p}$

Esquivel-Macías, C., 1996, Invertebrados del Paleozoico Tardío de las regiones de Olinalá, Gro. y Patlanoaya, Pue.: México, D.F., Universidad Nacional Autónoma de México, tesis de maestría, 82 p.

Esquivel-Macías, C., Ausich, W.I., Buitrón-Sánchez, B.E., Flores de Dios, A., 2000, Pennsylvanian and Mississippian pluricolumnal assemblages (Class Crinoidea) from southern Mexico and a new occurrence of a column with a tetralobate lumen: Journal of Paleontology, 74, 1187-1190.

Flores de Dios, A., Vachard, D., Buitrón-Sánchez, B.E., 2000, La cubierta sedimentaria Pérmica Superior (Formaciones Olinalá, Ihualtepec y Yododeñe) de los terrenos Mixteco y Zapoteco (resumen): GEOS, 20, 324-325.

Fries, C., Schmitter, E., Damon, P.E., Livingstone, D.E., 1962, Rocas precámbricas de edad Grenvilliana de la parte central de Oaxaca en el Sur de México: Boletín del Instituto de Geología UNAM, 64, 45-53.

Gluchowski, E., 2002, Crinoids from the Fammenian of the Holy Cross Mountains, Poland: Acta Palaeontologica Polonica, 47, 319-328.

Gluchowski, E., Racki, G., 2005, Disarticulated crinoids items from the Devonian and Carboniferous of north Devon, England: Proceedings of the Yorkshire Geological Society, 55, 161-172.

Landing, E., English, A., Keppie, J.D., 2010, Cambrian origin of all skeletalized metazoan phyla discovery of Earth's oldet bryozoans (Upper Cambrian, southern Mexico): Geology, 38, 547-550.

López-Lara, O., 2002, Crinoides (Echinodermata-Crinoidea) del Paleozoico Tardío de la región de Pemuxco, Hidalgo: Consideraciones estratigráficas y paleogeográficas: México, D.F., México, Universidad Nacional Autónoma de México, tesis de licenciatura, 80 p.

Moore, R.C., Jeffords, R.M., 1968, Classification and nomenclature of fossil crinoids based on studies of dissociated parts of their columns: The University of Kansas Paleontological Contributions, Serial Number 46, Article 9, 1-86.

Morón-Ríos, A., Perrilliat, M.C., 1988, Una especie nueva del género Griffithides Portlock (Arthropoda, Trilobita) del Paleozoico Superior de Oaxaca: Revista del Instituto de Geología UNAM, 7, 67-70.

Navarro-Santillán, D., Sour-Tovar, F., Centeno-García, E., 2002, Lower Mississippian (Osagean) brachiopods from the Santiago Formation, Oaxaca, Mexico: stratigraphic and tectonic implications: Journal of South American Earth Sciences, 15, 327-336.

Ortega-Gutierrez, F., Ruíz, J., Centeno-García, E., 1995, Oaxaquia, a Proterozoic microcontinent accreted to North America during late Paleozoic: Geology, 23, 1127-1130.

Pantoja-Alor, J., 1970, Rocas sedimentarias de la región centroseptentrional de Oaxaca, en Libro-Guía de la Excursión MéxicoOaxaca: México, D.F., Sociedad Geológica Mexicana, 67-84.

Quiroz-Barroso, S.A., Perrilliat, M.C., 1997, Pennsylvanian nuculoids (Bivalvia) from the Ixtaltepec Formation, Oaxaca, Mexico: Journal of Paleontology, 71, 400-407.

Quiroz-Barroso, S.A., Perrilliat, M.C., 1998, Pennsylvanian bivalves from the Ixtaltepec Formation, Mexico: Journal of Paleontology, 72, 1011-1024.
Quiroz-Barroso, S.A., Pojeta, J., Sour-Tovar, F., Morales-Soto, S., 2000, Pseudomulceodens: a Mississippian Rostroconch from Mexico: Journal of Paleontology, 74, 1184-1186.

Scheffler, S.M., Sequeira, F.A.C., 2007, Crinoidea da Formação Ponta Grossa (Devoniano, Bacia do Paraná), Brasil: Rio de Janeiro, Brasil, Arquivos do Museu Nacional, 65, 83-98.

Simon, P.R., Geizer, H.N., 1976, A pluricolumnal assemblage from the Ames Limestone in eastern Ohio: The Compass-Sigma Gamma Epsilon, 53, 85-106.

Sisova, E.N., 1988, Late Famennian crinoids of Central Kazakhstan: Ezhegodnik Vsesoyuznogo Paleontologiceskogo Obshchestva, $31,146-158$

Solís-Marín, F., Esquivel-Macías, C., Buitrón-Sánchez, B.E., 2004, Nuevos registros de placas columnares de crinoides (Echinodermata, Crinoidea) del Paleozoico Superior de México, algunas implicaciones paleobiogeográficas y paleoambientales: Coloquios de Paleontología, $54,15-24$.

Sour-Tovar, F., Martínez-Chacón, M.L., 2004, Braquiópodos chonetoideos del Carbonífero de México: Revista Española de Paleontología, $19,125-138$

Sour-Tovar, F., Quiroz-Barroso, S.A., 1989, Braquiópodos (Strophomenida) pensilvánicos de la Formación Ixtaltepec: Revista de la Sociedad Mexicana de Paleontología, 2, 5-17.

Sour-Tovar, F., Quiroz-Barroso, S.A., 1991, Icnofósiles paleozoicos de Nochixtlán, Oaxaca (resuemn), en Memorias del III Congreso Nacional de Paleontología: México, D.F., Sociedad Mexicana de Paleontología, $131 \mathrm{p}$.

Streng, M., Mellbin, B.B., Landing, E., Keppie, J.D., 2011, Linguliform brachiopods from the terminal Cambrian and Lowest Ordovician of the Oaxaquia Microcontinent (Southern Mexico): Journal of Paleontology, 85, 122-155.

Strimple, H.L., 1971, A Permian crinoid from Coahuila, Mexico: Journal of Paleontology, 45, 1040-1042.

Torres-Martínez, M.A., Sour-Tovar, F., Pérez-Huerta, A., 2008, Neospiriferinid brachiopods (Spiriferidae, Trigonotretidae) from Ixtaltepec Formation, Pennsylvanian of Oaxaca State, Southern Mexico: Fossils and Strata, 54, 157-166.

Vachard, D., Flores de Dios, A., Buitrón, B.E., 1997, Sur une nouvelle localité à fusulines du Wordien (Permien Supérieur) du Mexique: conséquences paléogéographiques: Geobios, 30, 361-370.

Velasco de León, P., Buitrón-Sánchez, B.E., 1992, Algunos crinoides (Echinodermata-Crinoidea) del Misisípico-Pensilvánico de San Salvador Patlanoaya, estado de Puebla: Revista de la Sociedad Mexicana de Paleontología, 5, 71-81.

Villaseñor-Martínez, A.B., Martínez-Cortés, A.M., Contreras y Montero, B., 1987, Bioestratigrafía del Paleozoico Superior de San Salvador Patlanoaya, Puebla, México: Revista de la Sociedad Mexicana de Paleontología, 1, 396-417.

Webster, G.D., Houck, K.J., 1998, Middle Pennsylvanian, late Atokanearly Desmoinesian echinoderms from an intermontane basin, the Central Colorado trough: Journal of Paleontology, 72, 1054-1072.

Manuscrito recibido: Febrero 28, 2011.

Manuscrito corregido recibido: Agosto 16, 2011.

Manuscrito aceptado: Agosto 20, 2011. 